\title{
CLOSED-LOOP CONTROL OF VORTEX SHEDDING IN A SEPARATED DIFFUSER USING AN INVERSE METHOD
}

\author{
Takao Suzuki, Tim Colonius, and Douglas G. MacMartin ${ }^{\dagger}$ \\ Division of Engineering and Applied Science \\ California Institute of Technology, Pasadena, California 91125
}

\begin{abstract}
We propose a closed-loop control algorithm for vortex shedding in a separated diffuser. We introduce pulses of zero-net-mass injection (consecutive blowing and suction) together with an inverse vortex imaging method. This method estimates the circulation of a vortex in the separated region based on pressure at a limited number of observer points at the wall. The closed-loop algorithm determines when to start the pulse so that the vortex is pinched off with a size which minimizes the stagnation pressure loss. We examine the proposed method in a simplified flow by performing direct numerical simulations of two-dimensional diffusers. In order to investigate the robustness of the closed-loop control algorithm, we impose high frequency acoustic disturbance upstream of the separation point. The disturbances significantly reduce the effectiveness of open-loop control compared to the case where no external disturbances are added. By using closed-loop control, however, performance is once again substantially recovered in the presence of disturbances.
\end{abstract}

\section{INTRODUCTION}

To control vortex shedding from a bluff body, a variety of closed-loop control methods has been studied. The main components of a control strategy may be separated into three parts: the sensor and flow identification, the control algorithm, and the actuation. Various issues related to the three components have been studied in past; in particular, suppression of vortex shedding from a cylinder has been extensively investigated as a model problem.

In early work (Ffowcs Williams \& Zhao 1989; Roussopoulos 1993; Park, et al. 1994; and others), algorithms were designed so that the motion at the peak vortex-shedding frequency was stabilized based

Copyright (c)2004 by Takao Suzuki, Tim Colonius, and Douglas G. MacMartin. Published by the American Institute of Aeronautics and Astronautics, Inc., with permission. on the phase information from sensors in the wake without attempting to identify the flow structures. More recently, reduced-order-modeling has been introduced to represent the flow dynamics (c.f. Glezer, et al. (1989) and Berkooz (1993) for general studies). For example, Gillies (1998) used the Proper Orthogonal Decomposition (POD) to describe the wake motion and suppressed vortex shedding in computations by placing sensors in the wake to identify the POD modes. Singh, et al. (2001) also used POD and performed simulations of optimal closed-loop control with the information about the full flow-field. Min \& Choi (1999) computationally demonstrated (sub)optimal closed-loop control based on the information only on the cylinder surface. All these methods, however, required substantial computation to identify the instantaneous flow structure and/or to solve dynamical equations; hence, they need to be simplified for real-time closed-loop control.

In addition to POD, other structure identification techniques, such as stochastic estimation and wavelet analysis, have been investigated (c.f. Bonnet, et al. 1998). In particular, Chang, et al. (1999), Naguib, et al. (2001), and Murray \& Ukeiley (2003) proposed to identify the flow structures using surface pressure. These statistical approaches required the information from full flow-field measurements as input. For real-time closed-loop control in practical configurations, however, we need a technique that only requires a limited number of sensor inputs and uses a computationally fast model of the dynamics.

Closed-loop control has also been applied to internal flows. Kwong \& Dowling (1994) successfully suppressed the unsteady motion at the peak frequency in diffusers by using mass injection; however, the pressure recovery was not improved. It has been documented in past that large-scale unsteadiness causes significant stagnation pressure loss in diffuser flows (Reneau, et al. 1967; and others). Periodic mass injection near the separation point (i.e. synthetic jet) was shown to enhance diffuser performance by Amitay, et al. (2002) and Narayanan \& Banaszuk(2003). 


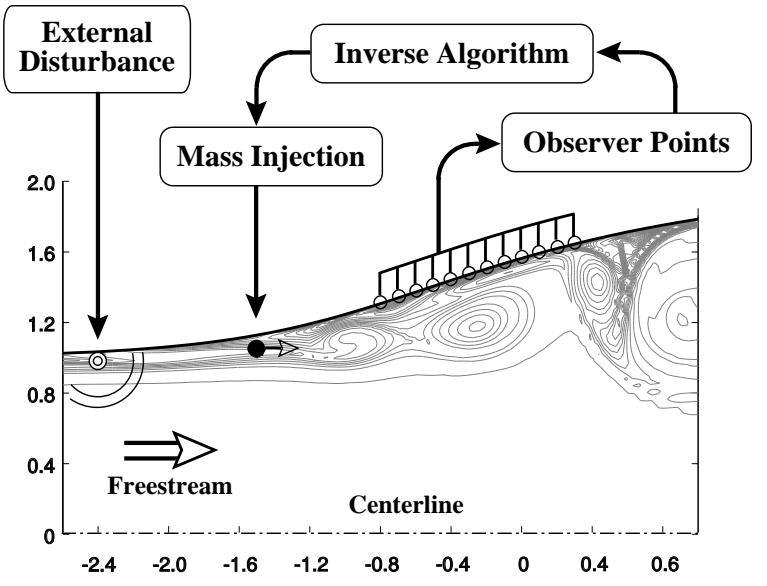

Figure 1: Schematic of the closed-loop control.

The mechanism of stagnation pressure loss due to vortex shedding was modeled by Suzuki, Colonius, \& Pirozzoli (2003). These studies have indicated that the key to reduce the stagnation pressure loss is to tune the forcing frequency so that the separated boundary layer is pinched off into vortices with an optimal size (i.e. circulation). The key change in the flow physics is a redistribution of the circulation rather than suppression of separation.

In this paper, we propose closed-loop control for vortex shedding and flow separation in a diffuser by introducing an inverse vortex imaging method (Suzuki \& Colonius 2003). Using least square optimization, this identification method can detect the position and circulation of a vortex based on pressure at a limited number of points on the wall; hence, unlike previous flow reconstruction techniques, no database of the flow-field is necessary a priori. For actuation we issue pulses of zero-net-mass injection (consecutive blowing and suction) in the boundary layer near the separation point. We first seek the actuation frequency that minimizes the stagnation pressure loss without external disturbances (i.e. open-loop control). From the optimal frequency case, we define a threshold circulation of pinchedoff vortices. In closed-loop control, we monitor the circulation of the growing vortex using the inverse imaging method, and activate a pulse only when the circulation exceeds the threshold value. Thus, a closed-loop is established on the time-scale of vortex shedding. A schematic diagram of the procedure is given in figure 1 . The proposed algorithm is examined by performing direct numerical simulation (DNS) in two-dimensions. The robustness of the closed-loop control is demonstrated against time-harmonic acoustic disturbances imposed in the boundary layer upstream of the separation point.

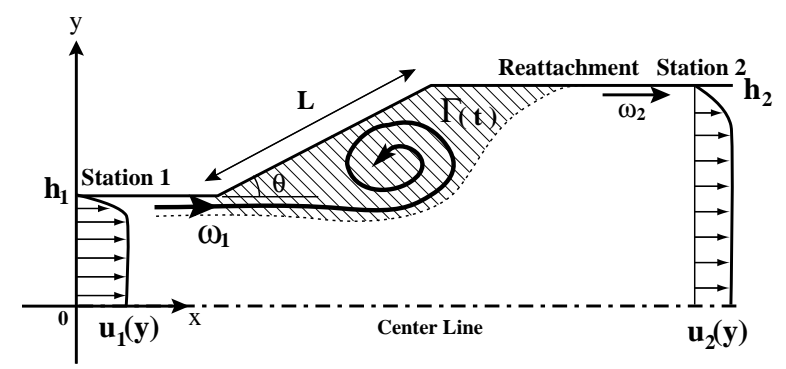

Figure 2: Simple two-dimensional diffuser model.

The outline of the paper is as follows: In the next section, the model of a planar diffuser (Suzuki, Colonius, \& Pirozzoli 2003) and the inverse vortex imaging method (Suzuki \& Colonius 2003) are reviewed. Next, the procedures of the numerical simulations including the models of the actuator and the external disturbance are presented. Subsequently, the closed-loop control is examined, and the results are discussed.

\section{MODEL OF VORTEX SHEDDING IN A PLANAR DIFFUSER}

First, we review the model of vortex shedding in a two-dimensional diffuser (Suzuki, Colonius, \& Pirozzoli 2003). This model explains the mechanisms of separation control using unsteady mass injection and motivates our strategies for closed-loop control.

\section{VORTEX SHEDDING FREQUENCY}

We consider an incompressible and inviscid flow and assume that the flow is symmetric about the centerline in a diffuser and transversely-sheared at the inlet and the exit (refer to figure 2 for the flow configuration). Accordingly, circulation accumulated per unit time due to the net vorticity flux is given by

$$
\frac{d \Gamma}{d t}=\frac{u_{1}^{2}(0)-u_{2}^{2}(0)}{2},
$$

where $u_{1}$ and $u_{2}$ are the inlet and exit velocities, respectively (the argument ' $(0)$ ' denotes that the velocity is evaluated at $y=0$, i.e. at the centerline).

We assume that the accumulated circulation forms a vortex in the separated region, and it is pinched off when the velocity of the outer radius of the vortex exceeds $\alpha u_{1}(0)$. Using Stokes' theorem, the time period of vortex shedding can be then calculated as

$$
T_{\text {shed }}^{M \rightarrow 0} \approx \frac{\alpha}{1-\lambda} \frac{2 \pi h_{1}\left(\frac{h_{2}}{h_{1}}\right)^{2}}{u_{1}(0)\left(1+\frac{h_{2}}{h_{1}}\right)},
$$




\section{AIAA Paper 2004-0577}

where $h_{1}$ and $h_{2}$ are the inlet and exit heights, respectively. $\lambda$ denotes the rate of absorption of circulation from the wall relative to (1). In unforced flows, the parameters were found to be $\alpha \approx 1$ and $\lambda \approx 0.1$ in the previous DNS study (Suzuki, Colonius, \& Pirozzoli 2003).

\section{STAGNATION PRESSURE LOSS}

Stagnation pressure loss can be now estimated using (2). Assuming that the vortex is radially symmetric, the stagnation pressure loss across a diffuser averaged in time and over the cross section can be obtained as

$$
\begin{aligned}
\left(\overline{p_{t 1}-p_{t 2}}\right)_{\mathrm{total}}^{M \rightarrow 0} & \approx \frac{\rho u_{1}^{2}}{2} \frac{\left(\frac{h_{2}}{h_{1}}-1\right)^{2}}{\left(\frac{h_{2}}{h_{1}}\right)^{2}} \\
\times & {\left[\frac{\overline{p_{w}^{*}}-\overline{p_{w}}}{\overline{p_{w}^{*}}-p_{1}}+\frac{\alpha \beta(1-\lambda)}{1-\alpha \kappa} \frac{\frac{h_{2}}{h_{1}}+1}{8}\right], }
\end{aligned}
$$

where

$$
\begin{aligned}
\overline{p_{w}} & \equiv \frac{1}{h_{2}-h_{1}} \int_{h_{1}}^{h_{2}} p_{\text {wall }} d y, \\
\overline{p_{w}^{*}} & \equiv p_{1}+\frac{\rho u_{1}^{2}}{2} \frac{\frac{h_{2}}{h_{1}}-1}{\frac{h_{2}}{h_{1}}}, \\
\beta & \equiv \frac{8 \pi}{\Gamma^{2}} \int_{-\infty}^{\infty} \int_{\infty}^{\tau} \int_{-\infty}^{\infty} v \omega d y d x d \tau,
\end{aligned}
$$

and

$$
\kappa\left(\frac{h_{2}}{h_{1}}, \frac{h_{3}}{h_{1}}\right) \equiv-\frac{\pi}{4}\left(\frac{h_{2}}{h_{1}}-1\right) \frac{1}{\tan \left(\pi \frac{h_{3}}{h_{1}} \frac{h_{1}}{h_{2}}\right)} .
$$

Here $h_{3}$ denotes the distance from the centerline to the vortex center and $\beta$ is defined so that $\beta=1$ for the Oseen vortex (Oseen 1912). $\overline{p_{w}^{*}}$ corresponds to the possible highest time-averaged pressure projected on the wall. The first term in (3) corresponds to the steady loss associated with the static pressure distribution on the wall in the separated region, and the second term to the unsteady loss caused by vortex shedding. The contribution from vortex shedding becomes comparable to the steady part as the convective velocity of the vortex decreases.

Although (3) is developed for natural vortex shedding, the formula can be applied to actively controlled cases by varying the parameters. In particular, if we pinch off vortices with a smaller size by periodically forcing the boundary layer, we can reduce

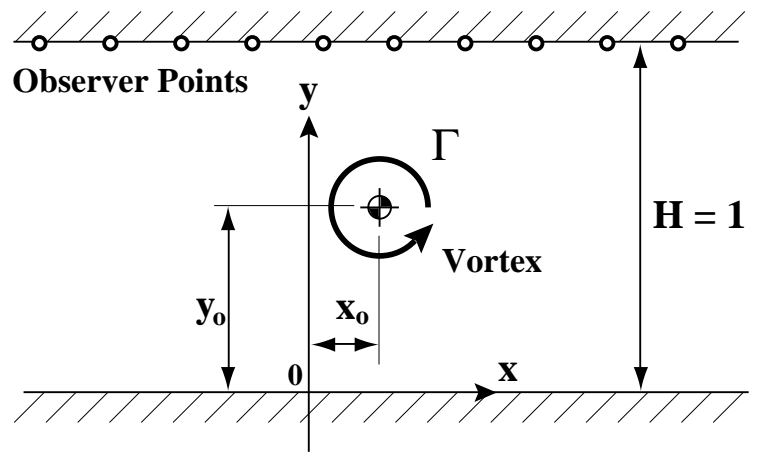

Figure 3: Schematic of the model problem.

$\alpha$ and suppress the substantial part of the unsteady stagnation pressure loss. Another key parameter, $\lambda$, also strongly depends on $\alpha$. This then leads to the observation which is the key to our control strategy: closed-loop control causing "optimal-sized" vortices to be pinched off should lead to minimum stagnation pressure loss.

\section{INVERSE IMAGING METHOD}

To exploit the connection between the actuation, the size of the shed vortices, and the stagnation pressure loss, we introduce an inverse vortex imaging method (Suzuki \& Colonius, 2003). This method can detect the position and circulation of a vortex (or vortices) from pressure histories at a limited number of points. We summarize this inverse method in this section.

\section{INVERSE ALGORITHM}

Suppose that we know the exact solution of the pressure field generated by a vortex in a flow as a function of its circulation, $\Gamma$, and position, $\left(x_{0}, y_{0}\right)$. We express this pressure as $p\left(\Gamma, x_{0}, y_{0} ; x, y\right)$, but eventually use a simple potential flow model as a surrogate for this pressure. We presume that time dependent pressure data are available at $N_{m}$ observer points $\left(1 \leq m \leq N_{m}\right)$ and express them as $q\left(t, x_{m}, y_{m}\right)$ (see figure 3). Now, we wish to find the set of parameters, $\Gamma$ and $\left(x_{0}, y_{0}\right)$, which best matches the pressure data to the solution by the following cost function of a least square type:

$$
\begin{aligned}
& J\left(t, \Gamma, x_{0}, y_{0}\right)= \\
& \sum_{m=1}^{N_{m}}\left[w_{1}\left(\Gamma, x_{0}, y_{0} ; q\right)\left|p\left(\Gamma, x_{0}, y_{0} ; x_{m}\right)-q\left(t, x_{m}\right)\right|^{2}\right. \\
& \left.+w_{2}\left(\Gamma, x_{0}, y_{0} ; \dot{q}\right)\left|\dot{p}\left(\Gamma, x_{0}, y_{0} ; x_{m}\right)-\dot{q}\left(t, x_{m}\right)\right|^{2}\right] .
\end{aligned}
$$




\section{AIAA Paper 2004-0577}

The first term in (8) primarily determines the position of the vortex in the streamwise direction and the second term that in the transverse direction. Here, we simply set the weight functions $w_{1}$ and $w_{2}$ so that the relative errors from both terms are equal and they depend only on the measured data (see Suzuki \& Colonius (2003) for the detail).

To minimize $J$, we differentiate (8) with respect to $\Gamma$. This must satisfy

$$
\frac{\partial J}{\partial \Gamma}=2 \sum_{m=1}^{N_{m}}\left[w_{1} \frac{\partial p}{\partial \Gamma}(p-q)+w_{2} \frac{\partial \dot{p}}{\partial \Gamma}(\dot{p}-\dot{q})\right]_{m}=0,
$$

where the subscript $m$ indicates that the quantity is evaluated at the $m^{\text {th }}$ observer position. Equation (9) gives the estimated circulation, $\Gamma^{*}$, if the vortex is located at $(x, y)=\left(x_{0}, y_{0}\right)$. Substituting $\Gamma^{*}$ into (8), we can compute the cost function at each point and map it on the $\left(x_{0}, y_{0}\right)$ domain. Thus, we create a "vortex image map" on which the local minimum of $J\left(t, \Gamma^{*}, x_{0}, y_{0}\right)$ indicates the vortex position. Figures 4 and 5 show an example of a vortex image map generated from DNS and the corresponding vorticity contour in a two-dimensional channel flow (Suzuki \& Colonius 2003). Here, $N_{m}=10$ observer points were used. The centroid of the vortex was detected within $0.03 H$ in this case.

\section{MODIFICATION OF ALGORITHMS FOR CLOSED-LOOP FLOW CONTROL}

In a plane channel, a simple potential flow solution can be used as a surrogate for $p\left(\Gamma, x_{0}, y_{0} ; x, y\right)$, as shown the previous examples (figures 4 and 5). In more complicated flow configurations, such as a diffuser in the present study, the potential solution for a point vortex may not be available in a simple formula. Instead, we approximate the potential solution by a family of simple functions with several parameters.

We consider a diffuser shape in which the coordinate in the streamwise direction is given by $\xi$ and that in the transverse direction by $\eta$ (see figure 6). By inspection, the position of the pressure minimum approximately gives the $\xi$ coordinate and the width of this pressure deficit strongly depends on the $\eta$ coordinate. In addition, as the circulation increases, the pressure deficit increases. From such observations, we construct an approximate potential solution on the upper wall using the following formula:

$\tilde{p}\left(a, b, c ; \Gamma, \xi_{0}, \eta_{0} ; \xi_{m}\right) \equiv \bar{p}\left(\xi_{m}\right)-\frac{a \Gamma^{c}}{b\left(1-\eta_{0}\right)} \mathrm{e}^{-\frac{\left(\xi_{m}-\xi_{0}\right)^{2}}{b^{2}\left(1-\eta_{0}\right)^{2}}}$,

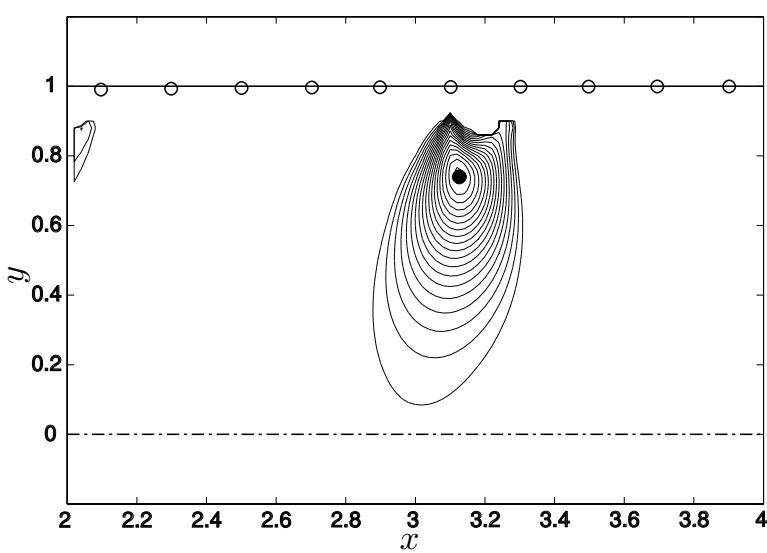

Figure 4: Image map of a vortex created using the inverse algorithm (Suzuki \& Colonius 2003). Contours of $-J$ corresponding to figure 5 are drawn. The detected position is denoted by $\bullet$, and the observer points are denoted by 0 .

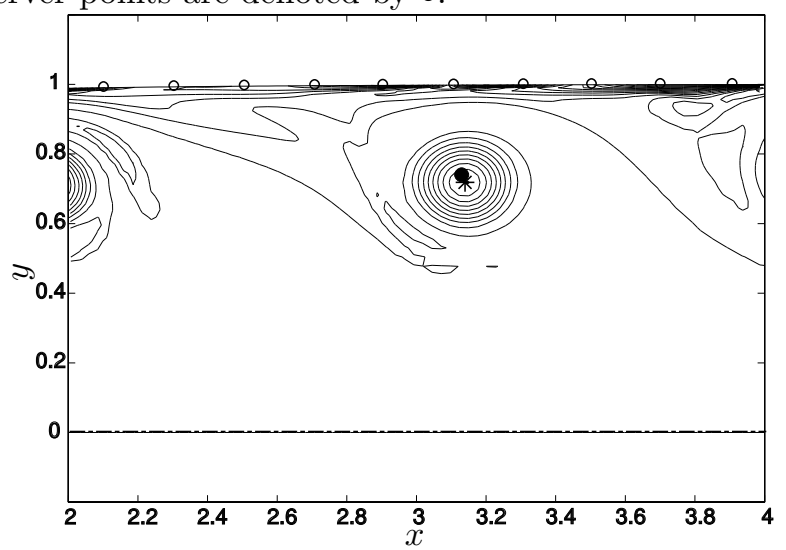

Figure 5: Detected vortex position using the inverse algorithm with vorticity contours (Suzuki \& Colonius 2003). The estimated circulation is $\Gamma=0.759$ as opposed to $\Gamma=0.909$ from DNS. The vortex centroid is denoted by $*$. The rest of the notation is the same as figure 4 .

where $\bar{p}\left(\xi_{m}\right)$ denotes the pressure at the $m^{\text {th }}$ observer when a vortex is absent.

To determine the free parameters, $a, b$, and $c$, we sample the solutions. By using the potential solutions for a point vortex with several different combinations of $\Gamma$ and $\left(\xi_{0}, \eta_{0}\right)$, we minimize the following cost function:

$$
\begin{aligned}
& \tilde{J}(a, b, c) \equiv \\
& \sum_{n=1}^{N_{n}} \varpi_{n} \sum_{m=1}^{N_{m}} \mid \tilde{p}\left(a, b, c ; \Gamma_{n},\left(\xi_{0}\right)_{n},\left(\eta_{0}\right)_{n} ; \xi_{m}, \eta_{m}\right) \\
& \quad-\left.p\left(\Gamma_{n},\left(\xi_{0}\right)_{n},\left(\eta_{0}\right)_{n} ; \xi_{m}, \eta_{m}\right)\right|^{2},
\end{aligned}
$$

where $N_{n}$ denotes the number of samples, $p$ is again 


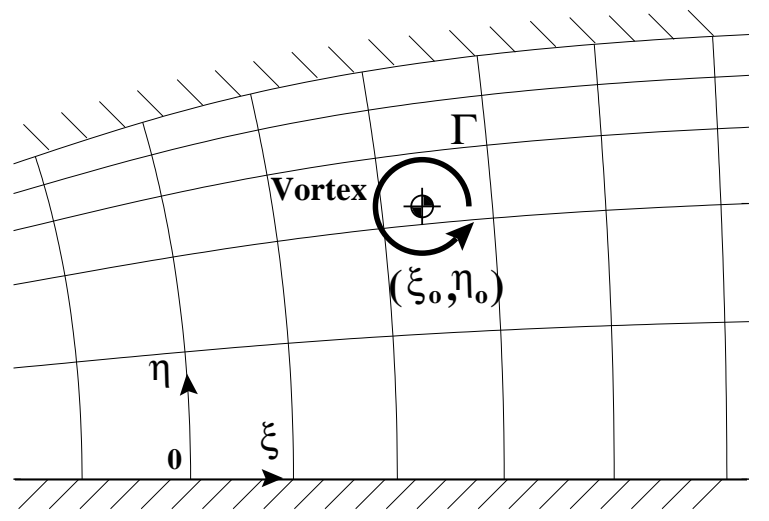

Figure 6: Coordinate system in a diffuser.

the potential solution, and the weight function is defined as $\varpi_{n} \equiv\left(\sum_{m=1}^{N_{m}} \mid p\left(\Gamma_{n},\left(\xi_{0}\right)_{n},\left(\eta_{0}\right)_{n} ; \xi_{m}, \eta_{m}\right)-\right.$ $\left.\left.\bar{p}\left(\xi_{m}\right)\right|^{2}\right)^{-1}$ for convenience. Determining $a, b$, and $c$, we can use $\tilde{p}$ instead of $p$ in (8) and follow the same procedure to create vortex image maps.

Note that the time derivative, $\tilde{\dot{p}}$, could be similarly approximated by some model function. However, to eliminate the iteration process for (9), we define the cost function without the time derivative term, i.e. the second term in (8). As a result, we can directly obtain the optimum circulation as

$$
\Gamma=\left[\frac{\sum_{m} \frac{a}{b\left(1-\eta_{0}\right)} \mathrm{e}^{-\frac{\left(\xi_{m}-\xi_{0}\right)^{2}}{b^{2}\left(1-\eta_{0}\right)^{2}}(\bar{p}-q)_{m}}}{\sum_{m} \frac{a^{2}}{b^{2}\left(1-\eta_{0}\right)^{2}} \mathrm{e}^{-2 \frac{\left(\xi_{m}-\xi_{0}\right)^{2}}{b^{2}\left(1-\eta_{0}\right)^{2}}}}\right]^{1 / c}
$$

This technique can substantially reduce computational time; hence, it is useful for real-time closedloop flow control.

When we keep track of the vortex position and circulation over time, we can take the flow dynamics into account by using the information from the previous time step; accordingly, we can suppress the random errors associated with each detection process. Based on the model of a diffuser described in the previous section, we state the dynamics of the vortex in the separated region as follows:

$$
\begin{aligned}
\frac{d \xi}{d t} & =\left[u_{\xi}+\frac{\Gamma}{4 h_{1} \tan (\pi \eta)}\right]\left|\frac{d \zeta}{d z}\right|, \\
\frac{d \eta}{d t} & =0 \\
\frac{d \Gamma}{d t} & =\frac{u_{1}^{2}-u_{2}^{2}}{2},
\end{aligned}
$$

where $z \equiv x+i y$ and $\zeta \equiv \xi+i \eta, u_{\xi}$ denotes the velocity of the potential solution without a vortex, and the conformal mapping is normalized so that $\frac{d \zeta}{d z} \rightarrow 1$ as $\xi \rightarrow-\infty$. The second term in (13) is calculated from the induced velocity of the mirrorimage vortices (c.f. section 1.5 in Saffman (1992)). Accordingly, we estimate the position and circulation of the vortex as

$$
\begin{aligned}
\xi_{i+1} & =r\left[\xi_{i}+\frac{d \xi}{d t} \Delta t\right]+(1-r) \xi_{i+1}^{\mathrm{inv}}, \\
\eta_{i+1} & =r\left[\eta_{i}+\frac{d \eta}{d t} \Delta t\right]+(1-r) \eta_{i+1}^{\mathrm{inv}}, \\
\Gamma_{i+1} & =r\left[\Gamma_{i}+\frac{d \Gamma}{d t} \Delta t\right]+(1-r) \Gamma_{i+1}^{\mathrm{inv}},
\end{aligned}
$$

where the subscript denotes the time step, and the weight factor, $r$, must be taken so that $r$. $\max \left[\right.$ eigenvalues of $\left.\left(I+\Delta t \frac{\partial \mathbf{u}}{\partial \mathbf{x}}\right)\right]<1$ (we took $r=$ 0.9 to satisfy this condition in this study). Here, the first term is given from the prediction based on the vortex dynamics and the second term from the detection using the inverse algorithm.

\section{NUMERICAL PROCEDURES}

To demonstrate the capabilities of the proposed control algorithm, we performed direct numerical simulations (DNS). Here, we summarize the numerical method and auxiliary procedures for implementing the closed-loop control. The full details of the numerical methods and a discussion of limitations are given in Suzuki, Colonius, \& Pirozzoli (2003).

\section{DIRECT NUMERICAL SIMULATION}

We solved the compressible Navier-Stokes equations to simulate unsteady, laminar diffuser flows in two-dimensions. We used the fourth order RungeKutta scheme for time marching and the sixth order Padé scheme for spatial derivatives of interior points with lower order closures (third and fourth order) at the inflow, exit, and wall boundaries. A two-dimensional diffuser shape (figure 7) was generated by conformal mapping. Among a variety of diffuser area ratios considered in the previous work, a representative case with an area ratio of $h_{2} / h_{1}=2$ is focused in the present study. $601 \times 151$ grid points were used in the $x$ and $y$ directions, respectively. A symmetry condition was assumed at $y=0$. Nonreflecting boundary conditions were imposed at both inflow and exit together with a "sponge" buffer zone (Freund 1997), in which the flow-field was forced to relax toward the initial solution.

The momentum thickness of the inlet laminar boundary layer was set to be $\delta_{b . l}=0.10 h_{1}$. The inlet Reynolds number and Mach number were $R e=$ 


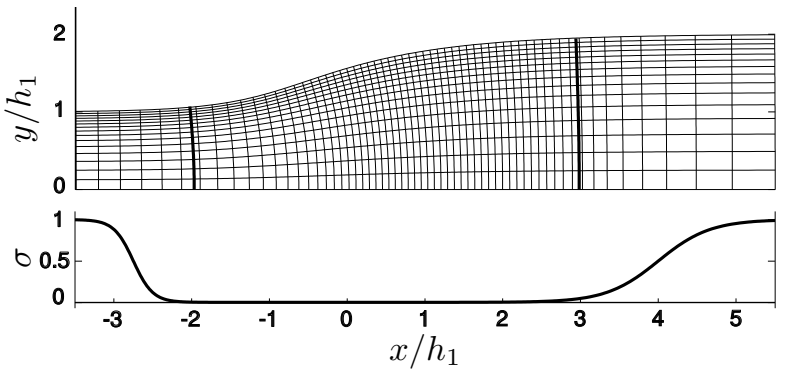

Figure 7: A computational grid on the top and the strength of the sponge term on the bottom. Every fifth grid point is shown, and the thicker lines denote the sections where the stagnation pressure loss was measured.

4000 and $M_{1}=0.4$, respectively. By varying parameters, we verified that the effects of compressibility and the Reynolds number on the large-scale flow patterns are minimal in a time-averaged sense in the previous study.

\section{MODELED ACTUATOR}

To simulate the actuator, we artificially forced the right hand side of the Navier-Stokes equations in a small region of the boundary layer. This idealized model is chosen primarily for computational simplicity, but the essential features of typical synthetic actuation are captured, as shown by Suzuki, Colonius, \& Pirozzoli (2003). The forcing terms were distributed using a narrow Gaussian shape function with deviations of $\sigma_{x} / h_{1}=0.08$ and $\sigma_{y} / h_{1}=0.01$ in the $x$ and $y$ directions, respectively. The center was positioned at $\left(x / h_{1}, y / h_{1}\right)=(-1.50,1.04)$.

The actuation velocity was

$u_{\text {jet }}(t)=0.9 a_{1} \frac{(f t)_{\mathrm{int}}-f_{\text {jet }} t}{\sigma_{t}} \exp \left[-\frac{\left(f_{\text {jet }} t-(f t)_{\mathrm{int}}\right)^{2}}{\sigma_{t}^{2}}\right]$,

where

$$
(f t)_{\text {int }} \equiv\left[\text { integer part of }\left(f_{\text {jet }} t-0.5\right)\right]+1 .
$$

Here $a_{1}$ is the speed of sound at the inlet, $\sigma_{t} u_{1} / h_{1}=$ $5.03 \times 10^{-2}$, and the subscript 'jet' denotes the value of the actuator. See figure 11 for the temporal wave form. Thus, as the forcing frequency increases, the effective blowing/suction period becomes correspondingly shorter. In the closed-loop cases, the actuation velocity profile was maintained, and the only the interval was modulated as mentioned before. Note that although we also tested an opposite phase of actuation, i.e. suction followed by blowing, it was found to be less effective than "blowing followed by suction" (about 10\% less in terms of improvement of stagnation pressure loss).

\section{EXTERNAL DISTURBANCES}

For the external disturbance, we imposed a time-harmonic acoustic source at the frequency of $f_{\text {T.H. }} h_{1} / u_{1}=0.517$ at $\left(x / h_{1}, y / h_{1}\right)=(-2.40,0.97)$ (refer to figure 1). This frequency is approximately five times as high as the natural vortex shedding frequency and was found to significantly deteriorate the performance of the open-loop forcing. The forcing was achieved by the terms on the right hand side of the Navier-Stokes equations within a small region in an analogous way to the actuator discussed above. The amplitude of pressure generated by this source was approximately $\left|\Delta p / p_{1}\right| \sim 3 \times 10^{-4}$ near the separation point. Refer to Suzuki \& Lele (2003) for the detailed procedures of the local acoustic forcing $\left(A_{p} \sim 2.5 \times 10^{-3}\right.$ in their $\left.(3.2)-(3.4)\right)$.

\section{INVERSE METHOD}

To measure pressure over time, we distributed 12 observer points on the wall slightly downstream of the separation point with a spacing of $0.1 h_{1}$ in the $x$ direction (see figure 8). By subtracting the freestream pressure given by the potential solution, we processed only the fluctuation part. Likewise, the freestream convective velocity, $u_{\xi}$ in (13), was calculated from the potential solution. The pressure data were sampled at the computation time step of $\Delta t u_{1} / h_{1}=3.2 \times 10^{-2}$. The target domain is depicted in figure 8 , and the resolution of detection was set to be $\Delta \xi=\Delta \eta=0.01$.

To define the approximated solution for a curved shape of the diffuser, the free parameters in (10) were determined from the potential solutions. Note that to use the fast algorithm, $c=2$ was chosen and only $a$ and $b$ were determined according to the method described before: Solutions at nine sample points (depicted in figure 8) times three values of circulation $\left(\Gamma /\left(u_{1} h_{1}\right)=0.333,0.667\right.$, and 1.333), i.e. a total of 27 samples, were were solved. Subsequently, the pressure profiles at the observer points were computed based on the unsteady Bernoulli's equation, and they were substituted into (11); as a result, $a=5.48 \times 10^{-3}, b=0.600$, and $c=2$ were determined.

Although we used the estimator-corrector and the fast algorithm to reduce uncertainty of prediction and computational cost, respectively, these modifications cause to deteriorate the accuracy of prediction. Figure 8 shows the comparison of the vortex trajectories: one obtained from DNS and the others 
AIAA Paper 2004-0577

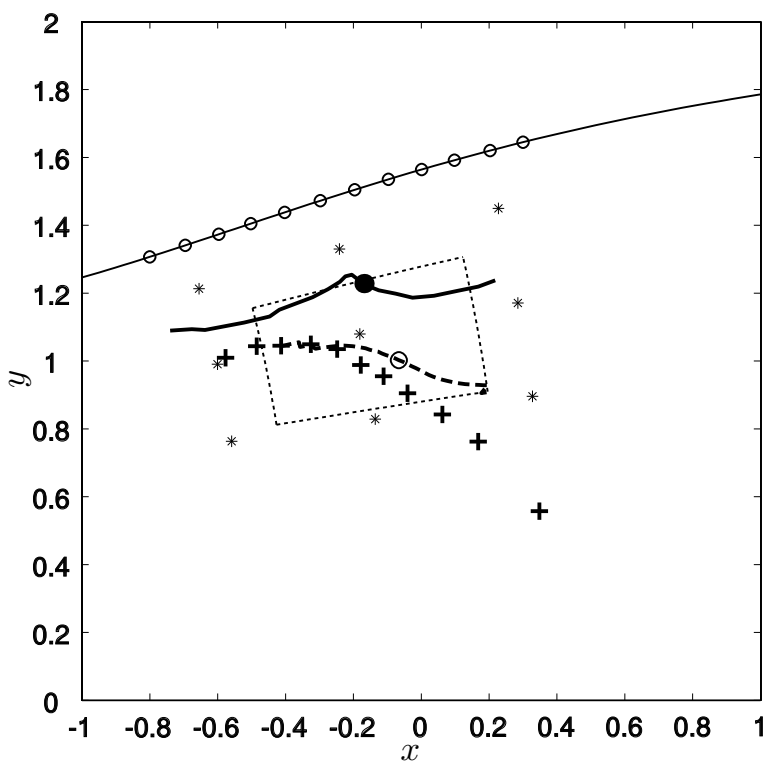

Figure 8: Points defined for the inverse algorithm: o on the wall, observer points; *, points where the sample solutions were computed. Vortex trajectories: —, local vorticity peaks given from DNS; +++ , the prediction using the fast inverse algorithm; - - - , that with the estimator-corrector. The position at which the pulse injection starts is indicated by $\bullet$ on the vorticity peak trajectory and by $\bigcirc$ on the estimated one. The target domain is denoted by $\cdots .$.

from the prediction with and without the estimatorcorrector. The accuracy substantially decreases as the vortex grows and moves downstream, mainly because its distribution is elongated in the flow direction. Since the simplified algorithm eliminates the time-dependence, the estimate in the transverse direction becomes poor in particular. However, as discussed later, the accuracy of the prediction is not crucial in the closed-loop algorithm as long as the trend of the error is consistent with the open-loop case.

\section{CLOSED-LOOP ALGORITHM}

The closed-loop control is designed so that a pulse is issued when the estimated circulation exceeds the threshold value $\left(\Gamma /\left(u_{1} h_{1}\right)=1.26\right.$ as discussed below). The estimator-corrector was activated only when the circulation was monotonically increasing (the minimum duration of $t u_{1} / h_{1}=0.16$ ) and the estimated position is inside the target domain. In addition, actuation was started only when the estimator-corrector was activated. To avoid staring actuation during the previous action, a minimum time interval of $t u_{1} / h_{1}=3.2$ was taken.

\section{RESULTS AND DISCUSSION}

\section{OPEN-LOOP CONTROL WITHOUT DISTURBANCE}

When the flow includes no external disturbances, periodic actuation (i.e. open-loop forcing) with sufficient amplitude induces frequency locking. In fact, the stagnation pressure profiles in figure 9 clearly exhibit it. To determine the optimal size of the separation vortex, we ran six different forcing frequencies with a fixed momentum coefficient $\left(C_{\mu} \equiv\right.$ $\left.\left(\overline{u_{\text {jet }}^{2}} h_{\text {jet }}\right) /\left(u_{1}^{2} h_{1}\right)=1.3 \times 10^{-3}\right)$ without external disturbances. Figure 10 shows that $f_{\text {jet }} h_{1} / u_{1}=0.20$ gives the minimum stagnation pressure loss. Accordingly, monitoring the blowing/suction velocity and the circulation of the vortex over time with the inverse imaging method in the optimal case, we determine the threshold value of circulation to be $\Gamma /\left(u_{1} h_{1}\right)=1.26$, at which the actuation should start (see figure 11). This information is used for closedloop control. We also note that the stagnation pressure loss is halved at the optimal frequency relative to the unforced case.

\section{SIMULATIONS WITH DISTURBANCE}

We now show the results for time-harmonic external disturbances. Figure 12 compares the stagnation pressure profiles between the open-loop and closedloop cases. In the open-loop case, the inflow disturbance disrupts frequency locking: The troughs in the exit stagnation pressure repeat with nearly the same interval, but their widths and depths vary. This indicates that the sizes of the convective vortices are not uniform. As a result, the stagnation pressure loss increases to $\overline{\Delta p_{t}} /\left(\rho u_{1}^{2} / 2\right)=0.167$ as opposed to $\overline{\Delta p_{t}} /\left(\rho u_{1}^{2} / 2\right)=0.123$ in the non-disturbance case (figure 9$)$. In particular, vortex paring causes significant stagnation pressure loss as reported by Suzuki, Colonius, \& Pirozzoli (2003) and Narayanan \& Banaszuk (2003).

When the closed-loop control is activated, frequency locking is nearly recovered, and the stagnation pressure loss is improved to $\overline{\Delta p_{t}} /\left(\rho u_{1}^{2} / 2\right)=$ 0.137. This effect is clearly observed in the phase relations between the actuator velocity and the estimated circulation in figure 13. Comparing with 11, phase locking is disrupted when the open-loop control is applied; in contrast, the closed-loop control is able to recovery frequency locking after a couple of transient cycles. Thus, the closed-loop control can adjust the forcing frequency to that of the external disturbance. It should be noted that although the estimate based on the fast inverse algorithm is not accurate, we expect that the actual circulation of 


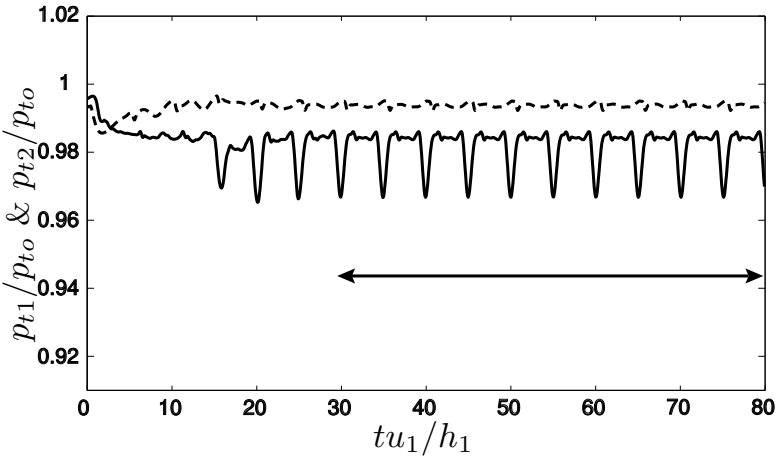

Figure 9: Comparison of stagnation pressure profiles in a non-disturbance operation. Stagnation pressure averaged over the inlet cross section $(x=-2)$ is denoted by --- , and that over the exit $(x=3)$ is - The forcing frequency is $f_{\text {jet }} h_{1} / u_{1}=0.2$ (the optimal).

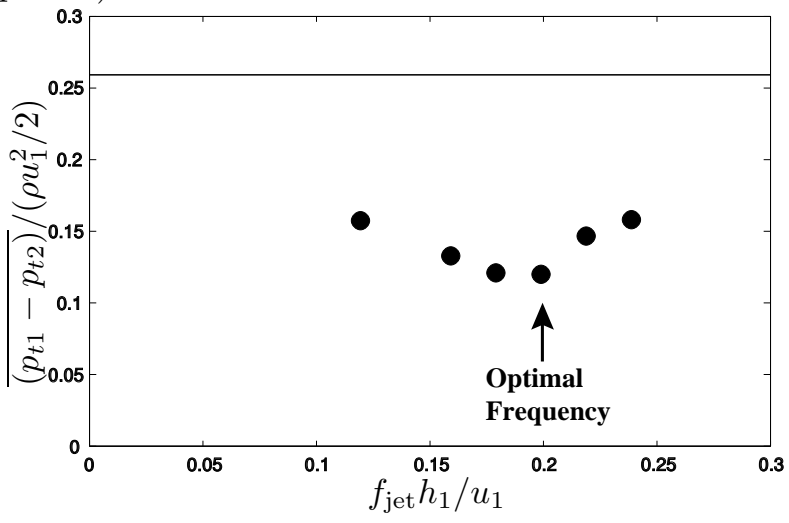

Figure 10: Stagnation pressure loss in nondisturbance operations. The horizontal line denotes the unforced case. The data were taken after the section averaged stagnation pressure loss became periodic as denoted by arrows in figure 9 .

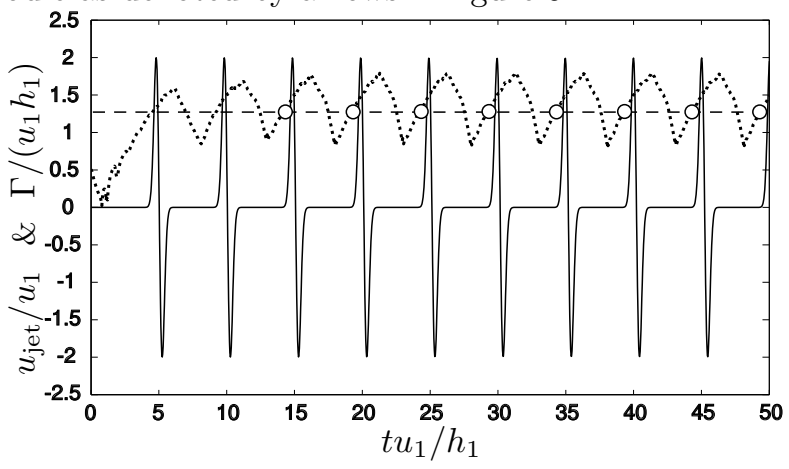

Figure 11: Phase relation between the injection velocity (denoted by - ${ }_{-}$) and the estimated circulation (denoted by $\cdots .$. ) of separation vortices at the optimal frequency $\left(f_{\text {jet }} h_{1} / u_{1}=0.2\right)$. The threshold value of circulation is denoted by 0 . the pinched-off vortices in the closed-loop operation is approximately equal to that in the case without the disturbances.

\section{CONCLUSIONS}

This study has demonstrated the capabilities of a proposed closed-loop control for vortex shedding in a diffuser using DNS. We have introduced an inverse imaging method which detects the circulation of a vortex growing near the separation point. We have designed the closed-loop algorithm so that zero-netmass injection near the separation point pinches off the vortex with a size that minimizes the stagnation pressure loss. The closed-loop control can almost recover frequency locking, which is disrupted when the external disturbances are added during openloop control. As a result, stagnation pressure loss is substantially improved.

This inverse method can extract an instantaneous flow-field only from information at a limited number of points on the boundary. Thus, it is relevant to various applications for closed-loop flow control, including detection of streamwise vortices from trailing edges or on a delta wing, and a large-scale vortex in a cavity. To capture more complicated flow structures or to improve the accuracy, we can introduce more parameters in the cost function although the convergence of optimization tends to become less stable and to require more computation.

Unlike optimal closed-loop algorithms proposed in past, the current technique only requires a simple phase relation in terms of one parameter, i.e. circulation. In the inverse-imaging process, however, the estimated position of the vortex is also available. Because several input parameters, such as the temporal wave-form of injection, are not fully optimized, we may be able to further improve the closed-loop control performance by investigating detailed flow physics of separation control.

Although we have performed the closed-loop control on the time-scale of vortex shedding, its frequency could be very high for practical flow configurations, and we must modulate the input parameters over several cycles or develop a closed-loop algorithm in the frequency domain. However, even in the frequency domain, the same idea should be applicable to reduce the stagnation pressure loss. Namely, counter to previous studies, this study indicates that we should design the control law so that the closedloop algorithm tries to concentrate the unsteady motions at an optimal frequency, rather than canceling disturbances at the natural peak frequency. It should be emphasized that the stagnation pressure 

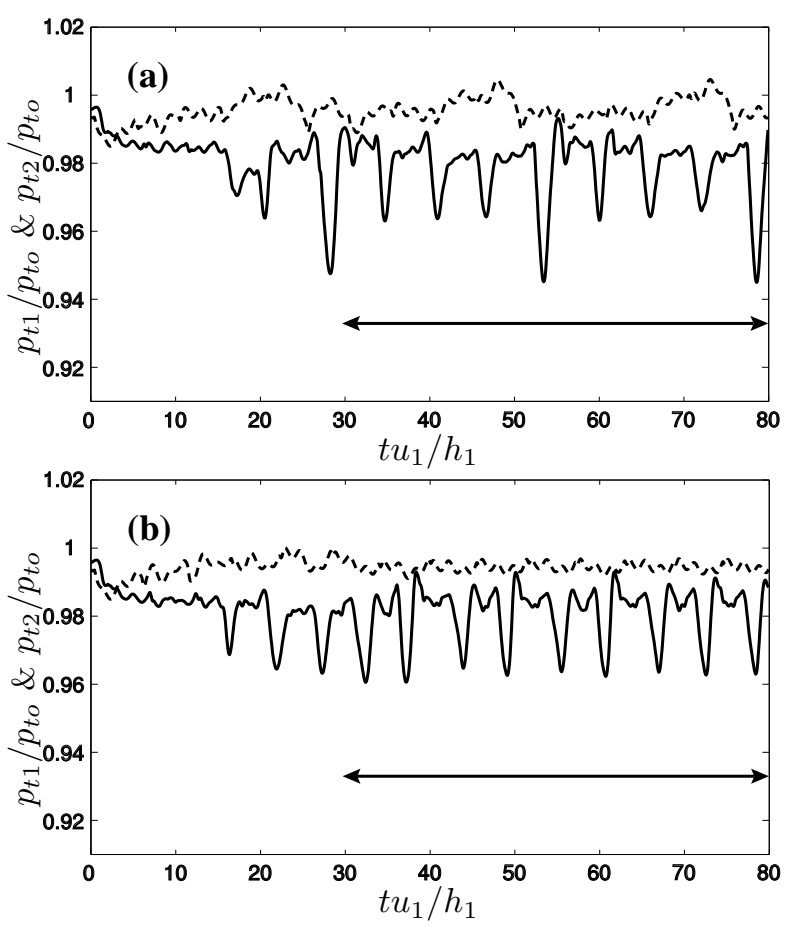

Figure 12: Comparison of stagnation pressure profiles when the time-harmonic disturbance is imposed: (a) open-loop control; (b) closed-loop control. Notation is the same as figure 9.
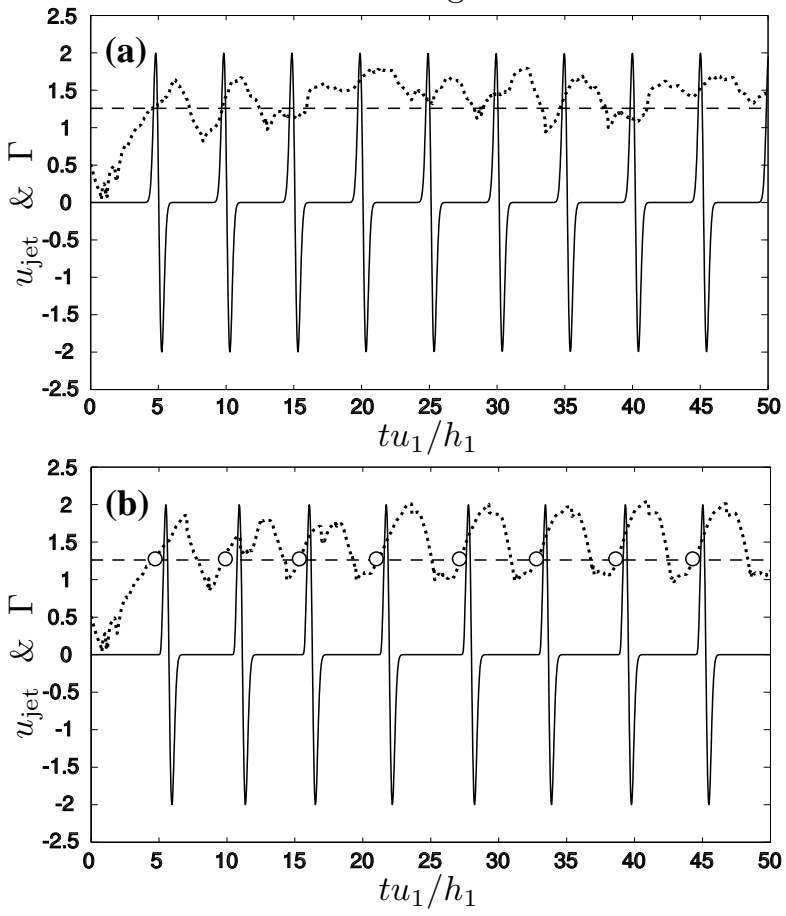

Figure 13: Phase relations between the actuator velocity and the estimated circulation when the timeharmonic disturbance is imposed: (a) open-loop control; (b) closed-loop control. Notation is the same as figure 11 . loss is most reduced when frequency locking is induced.

\section{$\underline{\text { ACKNOWLEDGMENTS }}$}

We would like to thank Prof. Hassan M. Nagib for many fruitful discussions on separation control.

\section{REFERENCES}

Bonnet, J. P., et al. 1998 Collaborative testing of eddy structure identification methods in free turbulent shear flows. Experiments in Fluids, 25, 197225 .

Berkooz, G., Holmes, P., \& Lumley, J. L. 1993 The proper orthogonal decomposition in the analysis of turbulent flows. Annu. Rev. Fluid Mech. 25, 539575 .

Chang, P. A., Piomelli, U., \& Blake, W. K. 1999 Relationship between wall pressure and velocity-field sources. Phys. Fluids, 11, no.11, 3434-3448.

Ffowcs Williams, J. E. \& Zhao, B. C. 1989 The active control of vortex shedding. J. Fluids Structs. 3, $115-122$.

Freund, J. B. 1997 Proposed inflow/outflow boundary condition for direct computation of aerodynamic sound. AIAA J. 35, no.4, 740-742.

Gillies, E. A. 1998 Low-dimensional control of the circular cylinder wake. J. Fluid Mech. 371, 157178.

Glezer, A., Kadioglu, Z., \& Pearlstein, A. 1989 Development of an extended proper orthogonal decomposition and its application to a time periodically forced plane mixing layer. Phys. Fluids A, 1, no.8, $1363-1373$.

Kwong, A. H. M. \& Dowling, A. P. 1994 Active boundary-layer control in diffusers. AIAA J. 32, no.12, 2409-2414.

Min, C. \& Choi, H. 1999 Suboptimal feedback control of vortex shedding at low Reynolds numbers. $J$. Fluid Mech. 401, 123-156.

Murray, N. E. \& Ukeiley, L. S. 2003 Estimation of the flowfield from surface pressure measurements in an open cavity. AIAA J. 41, no.5, 969-972.

Naguib, A. M., Wark, C. E., \& Juckenhöfel, O. 2001 Stochastic estimation and flow sources associated with surface pressure events in a turbulent boundary layer. Phys. Fluids, 13, no.9, 2611-2626. 
AIAA Paper 2004-0577

Narayanan, S. \& Banaszuk, A. 2003 Experimental study of a novel active separation control approach. AIAA Paper, AIAA 2003-0060.

Oseen, C. W. 1912 Über Wirbelbewegung in einer reibenden Flüssigkeit. Ark. f. Mat. Astron. Fys., 7,14 .

Park, D. S., Ladd, D. M., \& Hendricks, E. W. 1994 Feedback control of von Kármán vortex shedding behind a circular cylinder at low Reynolds numbers. Phys. Fluids, 6, no.7, 2390-2405.

Roussopoulos, K. 1993 Feedback control of vortex shedding at low Reynolds numbers. J. Fluid Mech. 248, 267-296.

Saffman, P. G. 1992 Vortex Dynamics, Cambridge University Press.

Singh, S. N., Myatt, J. H., Addington, G. A., Banda, S. \& Hall, J. K. 2001 Optimal feedback control of vortex shedding using proper orthogonal decomposition models. Trans. ASME: J. Fluids Engng. 123, 612-618.

Suzuki, T. \& Colonius, T. 2003 An inverse-imaging method for detection of a vortex in a channel. AIAA J. 41, no.9, 1743-1751.

Suzuki, T., Colonius, T., \& Pirozzoli, S. 2003 Vortex shedding in a two-dimensional diffuser: theory and simulation of separation control by periodic mass injection. submitted to J. Fluid Mech.

Suzuki, T. \& Lele, S. K. 2003 Green's functions for a source in a boundary layer: direct waves, channelled waves and diffracted waves. J. Fluid Mech. 477, 129-173. 